\title{
Spin-orbit coupling induced Mott transition in $\mathrm{Ca}_{2-x} \mathrm{Sr}_{x} \mathrm{RuO}_{4}(0 \leq \mathrm{x} \leq 0.2)$
}

\author{
Guo-Qiang Liu \\ Max-Planck-Institut für Festkörperforschung, D-70569 Stuttgart, Germany
}

(Dated: October 5, 2018)

\begin{abstract}
We propose a new mechanism for the paramagnetic metal-insulator transition in the layered perovskite $\mathrm{Ca}_{2-x} \mathrm{Sr}_{x} \mathrm{RuO}_{4}(0 \leq \mathrm{x} \leq 0.2)$. The LDA $+U$ approach including spin-orbit coupling is used to calculate the electronic structures. In $\mathrm{Ca}_{2} \mathrm{RuO}_{4}$, we show that the spin-orbit effect is strongly enhanced by the Coulomb repulsion, which leads to an insulating phase. When Ca is substituted by $\mathrm{Sr}$, the effective spin-orbit splitting is reduced due to the increasing bandwidth of the degenerate $d_{x z}$ and $d_{y z}$ orbitals. For $x=0.2$, the compound is found to be metallic. We show that these results are in good agreement with the experimental phase diagram.

PACS numbers: 71.30.+h, 71.15.Mb, 71.27.+a, 71.20.-b
\end{abstract}

The layered perovskite $\mathrm{Ca}_{2-x} \mathrm{Sr}_{x} \mathrm{RuO}_{4}$ (CSRO) has been intensely studied during recent years since this series of compounds exhibits a variety of interesting physical properties as a function of the $\mathrm{Sr}$ concentration $x \underline{\underline{1}-\underline{8}}$ $\mathrm{Sr}_{2} \mathrm{RuO}_{4}$ is a p-wave superconductor $\frac{1,9}{1}$ with a $\mathrm{K}_{2} \mathrm{NiF}_{4}$ type structure. The substitution of $\mathrm{Ca}$ for $\mathrm{Sr}$ causes the $\mathrm{RuO}_{6}$ octahedra to rotate, and start to tilt at $x=0.5 \cdot \frac{5}{-}$ Following with the structure distortion, CSRO undergoes a series of phase transition from a paramagnetic metal $(0.5<\mathrm{x}<2)$ to a magnetic metal $(0.2<\mathrm{x}<0.5)$, and finally to a Mott insulator $(0<\mathrm{x}<0.2) \stackrel{4}{\underline{4}}$ It is unusual that in the Mott insulating regime the metal-insulator transition temperature $\left(T_{\mathrm{MI}}\right)$ is higher than the Néel temperature $\left(T_{N}\right)$ of the antiferromagnetic $(\mathrm{AFM})$ phase, which shows that a paramagnetic (PM) insulating phase exists between these transition temperatures ${ }^{5,10,11}$ For pure $\mathrm{Ca}_{2} \mathrm{RuO}_{4}$, the PM insulating regime extends from $T_{N}=110 \mathrm{~K}$ to $T_{\mathrm{MI}}=357 \mathrm{~K}, 2,3.10$ This property makes $\mathrm{Ca}_{2-x} \mathrm{Sr}_{x} \mathrm{RuO}_{4}(0<\mathrm{x}<0.2)$ different from other AFM Mott insulators.

Recently, Qi et al $\stackrel{12}{=}$ found that the substitution of the lighter $\mathrm{Cr}$ for the heavier $\mathrm{Ru}$ strongly depresses $T_{\mathrm{MI}}$ in $\mathrm{Ca}_{2} \mathrm{Ru}_{1-y} \mathrm{Cr}_{y} \mathrm{O}_{4}(0<\mathrm{y}<0.13)$, which implies a possible influence of the relativistic spin-orbit (SO) coupling on the Mott transition as pointed out by the authors. It is well known that $\mathrm{SO}$ coupling plays an important role in $5 d$ transition-metal oxides. For example, Kim et al $\stackrel{13}{\underline{13}}$ found that $\mathrm{Sr}_{2} \mathrm{IrO}_{4}$ is a $J_{\text {eff }}=1 / 2$ Mott insulator, and they showed that the unusual insulating state can be explained by the combined effect of the SO coupling and Coulomb interaction. In the $4 d$ oxides, the importance of SO coupling is under debate. Mizokawa et al. $\frac{14}{\underline{\mathrm{ob}} \mathrm{ob}}$ served strong $\mathrm{SO}$ coupling in $\mathrm{Ca}_{2} \mathrm{RuO}_{4}$ from their photoemission experiment. Based this finding, they argued that the strong $\mathrm{SO}$ coupling in $\mathrm{Ca}_{2} \mathrm{RuO}_{4}$ would cause a complex electronic configuration. Theoretical studies revealed strong $\mathrm{SO}$ effects in $\mathrm{Sr}_{2} \mathrm{RuO}_{4}$ and $\mathrm{Sr}_{2} \mathrm{RhO}_{4}, \frac{15,16}{,}$ which seemingly support the photoemission experiment. However, Fang et al $\stackrel{17,18}{\underline{1}}$ reported an $\mathrm{LDA}+U$ study of $\mathrm{Ca}_{2} \mathrm{RuO}_{4}$. They found the AFM state has a rather simple configuration $x y^{\uparrow \downarrow} x z^{\uparrow} y z^{\uparrow}$ without much influence of the SO coupling. These seemingly inconsistent viewpoints raise a question: what role does the $\mathrm{SO}$ coupling play in CSRO?

In this paper we present electronic structure calculations for $\mathrm{Ca}_{2-x} \mathrm{Sr}_{x} \mathrm{RuO}_{4}$ using the LDA $+U$ method including the $\mathrm{SO}$ coupling. We show the combination of the $\mathrm{SO}$ coupling and Coulomb repulsion opens a band gap in $\mathrm{PM} \mathrm{Ca}_{2} \mathrm{RuO}_{4}$. The appearance of the Mott insulating phase is strongly dependent on the tilting of the $\mathrm{RuO}_{6}$ octahedra, which naturally explains the PM Mott transition in the experimental phase diagram. On the other hand, we find SO has much less influence on the AFM order. We show that these phenomena can be explained by a simple formalism.

All the calculations in this work were performed with the full-potential linear augmented plane wave (FLAPW) within the local-density approximation (LDA), as implemented in package WIEN2K $\underline{19}^{19}$ Two experimental structure ${ }^{\frac{5}{2}}$ were considered in this work. For $\mathrm{Ca}_{2} \mathrm{RuO}_{4}$, we used the structure at $180 \mathrm{~K}$, with the space group Pbca, lattice constant $\mathrm{a}=5.394, \mathrm{~b}=5.600$, and $\mathrm{c}=11.765$ $\AA .5 \frac{5}{}$ For $\mathrm{Ca}_{1.8} \mathrm{Sr}_{0.2} \mathrm{RuO}_{4}$, we used the experimental structure at $10 \mathrm{~K}$, but the substitution of $\mathrm{Sr}$ for $\mathrm{Ca}$ is only taken into account via the structural changes. $\mathrm{Ca}_{1.8} \mathrm{Sr}_{0.2} \mathrm{RuO}_{4}$ also has the space group Pbca but with lattice constant $\mathrm{a}=5.330, \mathrm{~b}=5.319$, and $\mathrm{c}=12.409 \AA .5$ For the AFM state, we considered the 'A-centered' mode $\mathrm{3}^{3}$ The LDA $+U$ calculations were performed with $U=3.0$ $\mathrm{eV}$, which is similar to the value used by Fang et al $\stackrel{17,18}{=}$ We will show that this $\mathrm{U}$ value can reproduce the measured band gap in $\mathrm{Ca}_{2} \mathrm{RuO}_{4}$.

In Fig. 1, we present our theoretical band structures for paramagnetic $\mathrm{Ca}_{2} \mathrm{RuO}_{4}$ using different approximations. The LDA band structure is well known 20.21: the bands crossing the Fermi level are from $\mathrm{Ru} t_{2 g}$ orbitals, containing four $d$ electrons. Our LDA band structure shown in Fig. 1a is consistent with the previous study $\underline{20}$ The inclusion of the SO coupling (Fig. 1b) only shows some slight changes on the band structure. This is not surprising since the $\mathrm{SO}$ coupling constant $\zeta$ in $\mathrm{Ca}_{2} \mathrm{RuO}_{4}$ is presumably similar to the one in $\mathrm{Sr}_{2} \mathrm{RuO}_{4}$, where it is only about $93 \mathrm{meV} \stackrel{15}{\underline{n}}$ The inclusion of Coulomb interaction (Fig. 1c) also shows little influence on the band structure since $\mathrm{U}$ does not break the orbital symmetry in the paramagnetic state. Surprisingly, the combined inter- 

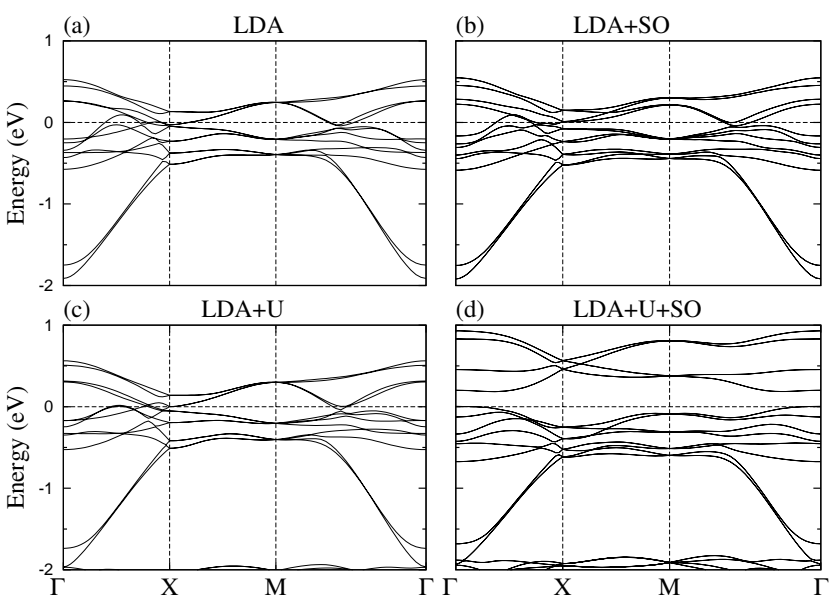

FIG. 1: Theoretical band structures for paramagnetic $\mathrm{Ca}_{2} \mathrm{RuO}_{4}$ using different approximations, (a) LDA, (b) $\mathrm{LDA}+\mathrm{SO}$, (c) $\mathrm{LDA}+U$, and (d) $\mathrm{LDA}+U+\mathrm{SO}$. The $\mathrm{LDA}+U$ and $\mathrm{LDA}+U+\mathrm{SO}$ band structures are calculated with $U=3.0$ $\mathrm{eV}$.

action of the SO coupling and Coulomb repulsion gives a very different band structure compared to the LDA, $\mathrm{LDA}+\mathrm{SO}$ or $\mathrm{LDA}+U$ results. The $\mathrm{LDA}+U+\mathrm{SO}$ band structure shows an insulating phase with a gap about 0.2 $\mathrm{eV}$ wide. The band gap obtained from the chosen $\mathrm{U}$ is in good agreement with the experimental data. $\frac{2,22}{2}$

Similar combined effect of the SO coupling and $U$ has

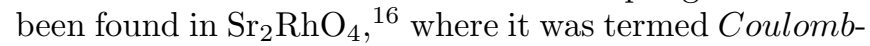
enhanced spin-orbit splitting. $\mathrm{Sr}_{2} \mathrm{RhO}_{4}$ has a similar crystal structure to $\mathrm{Ca}_{2-x} \mathrm{Sr}_{x} \mathrm{RuO}_{4}$, and it can be regarded as a two-band ( $x z$ and $y z)$ system since the $x y$ band is below the Fermi level due to the $\mathrm{RhO}_{6}$ rotation $\underline{23}, \underline{24}$ The simpler problem of $\mathrm{Sr}_{2} \mathrm{RhO}_{4}$ can help us to understand the $\mathrm{LDA}+U+\mathrm{SO}$ band structure of $\mathrm{Ca}_{2} \mathrm{RuO}_{4}$. In $\mathrm{Sr}_{2} \mathrm{RhO}_{4}$, the $\mathrm{SO}$ coupling splits the degenerate $x z$ and $y z$ bands to the higher $\chi_{ \pm 3 / 2}$ bands, and lower $\chi_{ \pm 1 / 2}$ bands, where

$$
\begin{array}{ll}
\chi_{3 / 2}=(x z+i y z) \uparrow, & \chi_{-3 / 2}=(x z-i y z) \downarrow \\
\chi_{1 / 2}=(x z+i y z) \downarrow, & \chi_{-1 / 2}=(x z-i y z) \uparrow .
\end{array}
$$

This splitting happens around the Fermi level, and therefore the occupancies of the $\chi_{ \pm 3 / 2}$ and $\chi_{ \pm 1 / 2}$ states are changed: $\left(n_{1 / 2}+n_{-1 / 2}\right)-\left(n_{3 / 2}+n_{-3 / 2}\right)=p>0$, where $n_{1 / 2}=n_{-1 / 2}$ and $n_{3 / 2}=n_{-3 / 2}$. When the Coulomb interaction is taken into account, the SO splitting is enhanced due to the different occupancies of the $\chi_{ \pm 3 / 2}$ and $\chi_{ \pm 1 / 2}$ states. The interplay the SO coupling and Coulomb interaction can be represented by an effective SO constant 16

$$
\zeta_{e f f}=\zeta+\frac{1}{2}(U-J) p,
$$

where $J$ is the Hund's coupling and $p$ is determined selfconsistently.

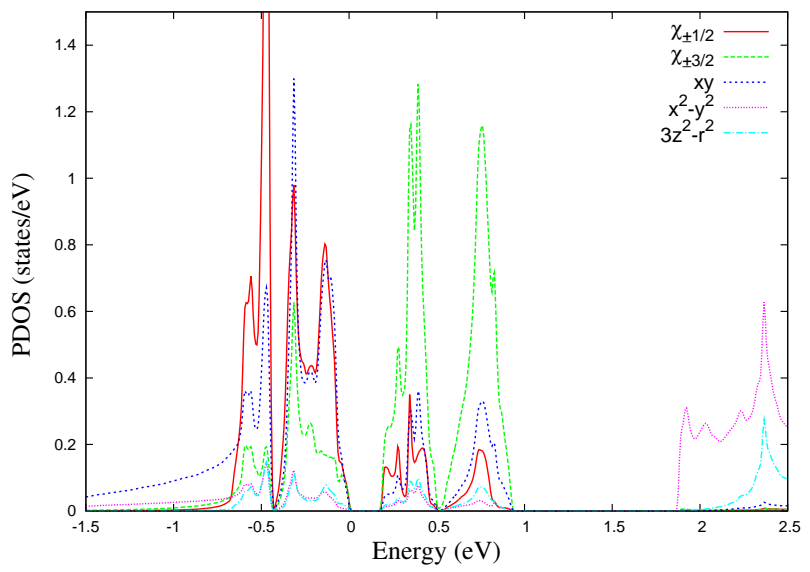

FIG. 2: Ru- $d$ PDOS for paramagnetic $\mathrm{Ca}_{2} \mathrm{RuO}_{4}$ calculated by $\mathrm{LDA}+U+\mathrm{SO}$.

The problem of $\mathrm{Ca}_{2} \mathrm{RuO}_{4}$ is more complicated than $\mathrm{Sr}_{2} \mathrm{RhO}_{4}$ since the $x y$ orbital is also involved. Fig. 2 presents the partial density of states (PDOS) for the Ru$d$ orbitals calculated by $\mathrm{LDA}+U+\mathrm{SO}$. Here we present the PDOS for the $\chi_{ \pm 3 / 2}$ and $\chi_{ \pm 1 / 2}$ orbitals instead of $x z$ and $y z$. The PDOS shows that the unoccupied $t_{2 g}$ bands $(0.2-0.9 \mathrm{eV})$ are dominated by the $\chi_{ \pm 3 / 2}$ states while the $\chi_{ \pm 1 / 2}$ states are nearly fully occupied. The well separated $\chi_{ \pm 3 / 2}$ and $\chi_{ \pm 1 / 2}$ states indicate a large effective spin-orbit splitting in $\mathrm{Ca}_{2} \mathrm{RuO}_{4}$. Therefore, A simple explanation for the PM Mott transition is that the Coulomb-enhanced spin-orbit splitting opens a gap between the $\chi_{ \pm 3 / 2}$ and $\chi_{ \pm 1 / 2}$ bands, leading to an insulating phase with two holes residing on the $\chi_{ \pm 3 / 2}$ orbitals. In this explanation, the $x y$ state is assumed to be fully occupied. However, in the experimental structure, the $x y$, $x z$ and $y z$ orbitals hybridize with each other due to the structural distortion. As may be seen, the weight of the $x y$ state in the unoccupied $t_{2 g}$ bands is not small as shown in Fig. 2, The relative hole population shown in Fig. 2 is $x y: \chi_{ \pm \frac{1}{2}}: \chi_{ \pm \frac{3}{2}}=19: 13: 68$, while this ratio is $21: 39.5: 39.5$ within LDA approximation. This shows that the inclusion of SO and U hardly changes the occupancy of the $x y$ orbital. We may conclude that the band gap is mainly due to the splitting of $\chi_{ \pm 3 / 2}$ and $\chi_{ \pm 1 / 2}$ orbitals although the $x y$ orbital is also involved in the Mott transition.

Experimental research has found that the Mott transition in CSRO is accompanied by an structural phase transition from the high temperature $L$-Pbca phase to the low temperature $S$-Pbca phase, $\stackrel{5,11}{=}$ where L (S) indicates a long (short) $c$-axis. The phase transition temperature $T_{S}$ is a function of Sr concentration $x$, which decreases from $357 \mathrm{~K}$ at $x=0$ to $0 \mathrm{~K}$ at $x \sim 0.2^{5.11}$. For $x \geq 0.2$, CSRO is metallic and only has the $L-P b c a$ phase. As indicated by Friedt et al.,$\frac{5}{,}$, the structural transition from the $L-P b c a$ to $S$-Pbca phase is characterized by an increase in the tilting angle of $\mathrm{RuO}_{6}$ octahedra. We will show that the tilting angle of $\mathrm{RuO}_{6}$ plays an important role in the Mott transition. To illuminate the 


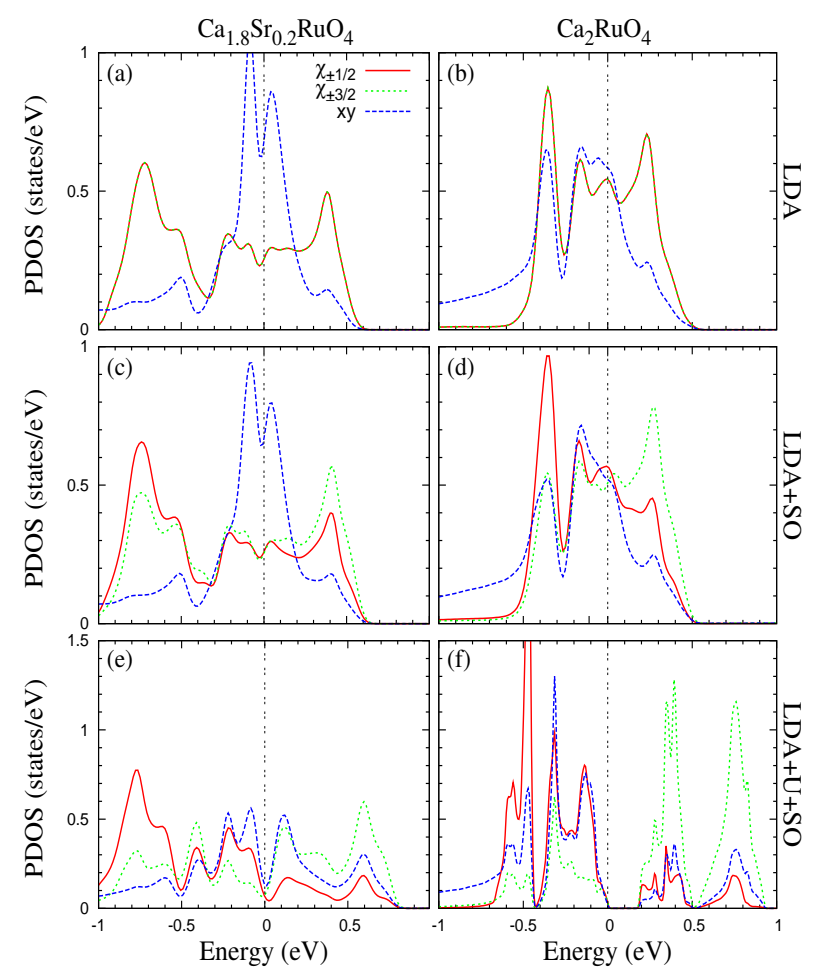

FIG. 3: Ru- $t_{2 g}$ PDOS for paramagnetic state using different approximations. The left panels are for $\mathrm{Ca}_{1.8} \mathrm{Sr}_{0.2} \mathrm{RuO}_{4}$, and right panels for $\mathrm{Ca}_{2} \mathrm{RuO}_{4}$.

relation between the Mott transition and the structural phase transition, we apply the $\mathrm{LDA}+U+\mathrm{SO}$ calculation to $\mathrm{Ca}_{1.8} \mathrm{Sr}_{0.2} \mathrm{RuO}_{4}$.

Fig. 3 presents our calculated PDOS for $x=0.2(L-$ $P b c a)$ and $x=0(S-P b c a)$. Fig. 3a and $3 b$ show the LDA PDOS for $x=0.2$ and $x=0$. The $x z / y z$ bandwidth is about $1.6 \mathrm{eV}$ in $\mathrm{Ca}_{1.8} \mathrm{Sr}_{0.2} \mathrm{RuO}_{4}$, and it is reduced to 1.1 $\mathrm{eV}$ in $\mathrm{Ca}_{2} \mathrm{RuO}_{4}$. The narrower $x z / y z$ band in $\mathrm{Ca}_{2} \mathrm{RuO}_{4}$ is due to its larger tilting angle. The tilting angle is about $12^{\circ}$ in $\mathrm{Ca}_{2} \mathrm{RuO}_{4}$, and it is $6^{\circ}$ in $\mathrm{Ca}_{1.8} \mathrm{Sr}_{0.2} \mathrm{RuO}_{4} \stackrel{\underline{\underline{5}}}{\text { The }}$ tilting of the in-plane $\mathrm{Ru}-\mathrm{O}$ can significantly reduces the interaction between $\mathrm{Ru}-d_{x z / y z}$ and $\mathrm{O}-p_{z}$. Consequently, the $x z / y z$ bandwidth decreases from $x=0.2$ to $x=0$, while the $x y$ bandwidth is less influenced. With the narrower $x z / y z$ band, $\mathrm{Ca}_{2} \mathrm{RuO}_{4}$ shows much higher $x z / y z$ PDOS around the Fermi level than $\mathrm{Ca}_{1.8} \mathrm{Sr}_{0.2} \mathrm{RuO}_{4}$. Fig. $3 \mathrm{c}$ and $3 \mathrm{~d}$ show the LDA+SO PDOS for $x=0.2$ and $x=0$. As may be seen, the occupancy difference between the $\chi_{ \pm 3 / 2}$ and $\chi_{ \pm 1 / 2}$ states is larger in $\mathrm{Ca}_{2} \mathrm{RuO}_{4}$ than in $\mathrm{Ca}_{1.8} \mathrm{Sr}_{0.2} \mathrm{RuO}_{4}$. This is understandable if we consider the higher PDOS in $\mathrm{Ca}_{2} \mathrm{RuO}_{4}$. Eq. (1) shows the effective SO splitting is proportional to the occupancy difference $p$. Then the lager occupancy difference $p$ in $\mathrm{Ca}_{2} \mathrm{RuO}_{4}$ will cause larger $\mathrm{SO}$ splitting when Coulomb interaction is taken into account. This is confirmed by the LDA $+U+\mathrm{SO}$ PDOS shown in Fig. 3e and 3f. Using Eq. (1), we get $\zeta_{e f f}=0.9 \mathrm{eV}$ for $\mathrm{Ca}_{2} \mathrm{RuO}_{4}$, and $0.6 \mathrm{eV}$ for $\mathrm{Ca}_{1.8} \mathrm{Sr}_{0.2} \mathrm{RuO}_{4}$. The larger $\mathrm{SO}$ splitting in $\mathrm{Ca}_{2} \mathrm{RuO}_{4}$

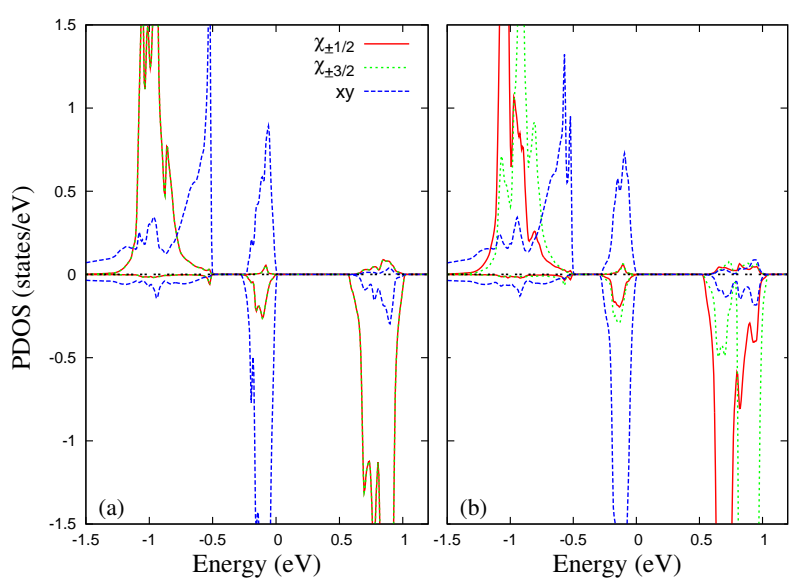

FIG. 4: Ru- $t_{2 g}$ PDOS for AFM $\mathrm{Ca}_{2} \mathrm{RuO}_{4}$ using different approximations, (a) $\mathrm{LDA}+U$, and (b) $\mathrm{LDA}+U+\mathrm{SO}$.

leads an insulating phase, while $\mathrm{Ca}_{1.8} \mathrm{Sr}_{0.2} \mathrm{RuO}_{4}$ remains metallic. Therefore, we have shown that the PM Mott transition in CSRO can be explained by the interplay of SO coupling, Coulomb interaction and structural distortion.

CSRO is a single-layer system, where the $t_{2 g}$ bands are split into the singly degenerate $x y$ band and doubly degenerate $x z / y z$ bands. The bare SO coupling mainly influences the degenerate bands since Ru has a moderate SO constant. Therefore, the SO induced Mott transition is strongly orbital dependent. Fig. 3 indicates that the Mott transition in PM CSRO is driven by the narrowing of the $x z$ and $y z$ bands, while the $x y$ orbital plays a less important role. This strong orbital-dependence could also be seen from Eq. (1). The bare SO parameter $\zeta$ is constant for each orbital, but the Coulomb enhanced SO parameter $\zeta_{e f f}$ is a function of orbital occupancies. This suggests that the strong orbital-dependence is an inherent feature of the SO induced Mott transition.

Our calculation has shown that the PM insulating phase of CSRO originates in the strong effective SO splitting. This picture supports the photoemission measure-

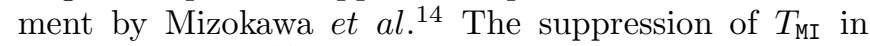
$\mathrm{Ca}_{2} \mathrm{Ru}_{1-y} \mathrm{Cr}_{y} \mathrm{O}_{4}{ }^{12}$ can also be understood within this picture. Cr has a much smaller atomic SO constant than $\mathrm{Ru}$ due to its smaller mass. And therefore the substitution of $\mathrm{Cr}$ for $\mathrm{Ru}$ will reduce the $\mathrm{SO}$ splitting, leading to the observed decrease of $T_{\mathrm{MI}}$.

As mentioned above, Fang et al $\underline{17,18}$ found that SO coupling has no much influence on the electronic configuration. They however pointed out that the photoemission measurement was done above the Néel temperature, while they applied the $\mathrm{LDA}+U$ method to the low temperature AFM state. To clarify if the SO coupling is less important in AFM state, we apply the $\mathrm{LDA}+U$ and $\mathrm{LDA}+U+\mathrm{SO}$ calculation to AFM $\mathrm{Ca}_{2} \mathrm{RuO}_{4}$. Our $\mathrm{LDA}+U$ calculation gives a magnetic moment of $m_{R u}=1.25 \mu_{B}$, which is consistent with Fang et al.'s calculation $\frac{17}{}$; while $\mathrm{SO}$ reduces the moment to 
$1.21 \mu_{B}$, showing a weak SO effect. The AFM PDOS for $\mathrm{Ca}_{2} \mathrm{RuO}_{4}$ are presented in Fig. 4. In contrast to the PM state, Fig. 4 shows that there is no Coulombenhanced SO splitting in the AFM state. The relative weak SO splitting in the AFM state can be explained by Eq. (1). The $\mathrm{LDA}+U$ calculation produces an insulating phase for AFM $\mathrm{Ca}_{2} \mathrm{RuO}_{4}$ as shown in Fig. 4a. Since there is no density of states around the Fermi level, SO coupling can not change the orbital occupancies, which gives $p=0$. Then we get $\zeta_{\text {eff }}=\zeta$, showing no enhanced $\mathrm{SO}$ splitting. It is noticeable that our calculations give an AFM ground state for $\mathrm{Ca}_{2} \mathrm{RuO}_{4}$, which is consistent with the experimental phase diagram. $\underline{\underline{5}}$

Comparing the PM and AFM insulating phases in CSRO, we may find that the two kinds of Mott transition are similar. They both have an interaction to break the orbital symmetry. The interaction is SO coupling in the PM state and spin polarization in the AFM state. The breaking of orbital symmetry lifts the degenerate bands and changes the orbital occupancies. When the Coulomb interaction is taken into account, the orbital splitting, which is SO splitting in the PM state or exchange splitting in the AFM state, is enhanced. If the enhanced splitting is large enough, it will lead to an in- sulating phase. The PM-AFM transition at $T_{N}$ can be regarded as the competition of the Coulomb-enhanced SO splitting and the Coulomb-enhanced exchange splitting. In the AFM state, the SO enhancement is quenched by the large exchange splitting, which causes the very different electronic configuration from the PM state.

In summary, we have applied $\mathrm{LDA}+U+\mathrm{SO}$ calculations to CSRO. We find the Coulomb enhanced SO splitting produces an insulating phase in $\mathrm{PM} \mathrm{Ca}_{2} \mathrm{RuO}_{4}$. This finding is consistent with the photoemission experiment, and also explains the recent experiment on $\mathrm{Ca}_{2} \mathrm{Ru}_{1-y} \mathrm{Cr}_{y} \mathrm{O}_{4}$. We show that the SO induced Mott transition in CSRO is driven by the change of the $x z / y z$ bandwidth. For $x=0.2$, the compound is found to be metallic. On the other hand, we find that $\mathrm{SO}$ coupling has much less influence on the AFM state, which is in agreement with the previous $\mathrm{LDA}+U$ study. The above picture shows that $\mathrm{SO}$ coupling plays a very subtle role in the correlated systems. The interplay of SO coupling, electron correlation and crystal structure distortion would cause very rich physical phenomena.

The author gratefully acknowledges Ove Jepsen for helpful discussions and useful comments.
${ }^{1}$ Y. Maeno, H. Hashimoto, K. Yoshida, S. Nishizaki, T. Fujita, J. G. Bednorz, and F. Lichtenberg, Nature (London) 372, 532 (1994).

2 G. Cao, S. McCall, M. Shepard, J. E. Crow, and R. P. Guertin, Phys. Rev. B 56, R2916 (1997).

3 M. Braden, G. Andre, S. Nakatsuji, and Y. Maeno, Phys. Rev. B 58, 847 (1998).

${ }^{4}$ S. Nakatsuji and Y. Maeno, Phys. Rev. Lett. 84, 2666 (2000).

5 O. Friedt, M. Braden, G. Andre, P. Adelmann, S. Nakatsuji, and Y. Maeno, Phys. Rev. B 63, 174432 (2001).

6 O. Friedt, P. Steffens, M. Braden, Y. Sidis, S. Nakatsuji, and Y. Maeno, Phys. Rev. Lett. 93, 147404 (2004).

7 M. Kriener, P. Steffens, J. Baier, O. Schumann, T. Zabel, T. Lorenz, O. Friedt, R. Muller, A. Gukasov, P. G. Radaelli, P. Reutler, A. Revcolevschi, S. Nakatsuji, Y. Maeno, and M. Braden, Phys. Rev. Lett. 95, 267403 (2005).

8 E. Gorelov, M. Karolak, T. O. Wehling, F. Lechermann, A. I. Lichtenstein, and E. Pavarini, Phys. Rev. Lett. 104, 226401 (2010).

9 A. P. Mackenzie and Y. Maeno, Rev. Mod. Phys. 75, 657 (2003).

10 S. Nakatsuji, S. Ikeda, and Y. Maeno, J. Phys. Soc. Jpn. 66, 1868 (1997).

11 C. S. Alexander, G. Cao, V. Dobrosavljevic, S. McCall, J. E. Crow, E. Lochner, and R. P. Guertin, Phys. Rev. B 60, R8422 (1999).

12 T. F. Qi, O. B. Korneta, S. Parkin, L. E. De Long, P. Schlottmann, and G. Cao Phys. Rev. Lett. 105, 177203
(2010).

13 B. J. Kim, Hosub Jin, S. J. Moon, J.-Y. Kim, B.-G. Park, C. S. Leem, Jaejun Yu, T. W. Noh, C. Kim, S.-J. Oh, J.-H. Park, V. Durairaj, G. Cao, and E. Rotenberg, Phys. Rev. Lett. 101, 076402 (2008).

14 T. Mizokawa, L. H. Tjeng, G. A. Sawatzky, G. Ghiringhelli, O. Tjernberg, N. B. Brookes, H. Fukazawa, S. Nakatsuji, and Y. Maeno, Phys. Rev. Lett. 87, 077202 (2001).

15 M. W. Haverkort, I. S. Elfimov, L. H. Tjeng, G. A. Sawatzky, and A. Damascelli, Phys. Rev. Lett. 101, 026406 (2008).

16 Guo-Qiang Liu, V. N. Antonov, O. Jepsen, and O. K. Andersen, Phys. Rev. Lett. 101, 026408 (2008).

17 Zhong Fang, Naoto Nagaosa, and Kiyoyuki Terakura, Phys. Rev. B 69, 045116 (2004).

18 J. H. Jung, Z. Fang, J. P. He, Y. Kaneko, Y. Okimoto, and Y. Tokura, Phys. Rev. Lett. 91, 056403 (2003).

19 P. Blaha et al., Computer code WIEN2K, TU Wien, Vienna, 2001.

${ }^{20}$ L. M. Woods, Phys. Rev. B 62, 7833 (2000).

21 Z. Fang and K. Terakura, Phys. Rev. B 64, R020509 (2001).

22 A. V. Puchkov, M. C. Schabel, D. N. Basov, T. Startseva, G. Cao, T. Timusk, and Z.-X. Shen, Phys. Rev. Lett. 81, 2747 (1998).

23 B. J. Kim, Jaejun Yu, H. Koh, I. Nagai, S. I. Ikeda, S.-J. Oh, and C. Kim, Phys. Rev. Lett. 97, 106401 (2006).

24 Eunjung Ko, B. J. Kim, C. Kim, and Hyoung Joon Choi, Phys. Rev. Lett. 98, 226401 (2007). 